\title{
Industrial Learning Curves: Series Production of the LHC Main Superconducting Dipoles
}

\author{
P. Fessia, F. Regis, and L. Rossi
}

\begin{abstract}
By mid August 2006, 1160 of the 1232 of LHC main dipoles have been delivered to CERN by the three suppliers in charge of the production. The training of the staff, mostly hired just for this manufacture, and the improvement of the procedures with the acquired experience, naturally decrease the time necessary for the assembly of a unit. The aim of this paper is to apply methodologies like the cost-based learning curves and the time-based learning curves to the LHC Main Dipole production comparing the estimated learning percentage to the ones experienced in other industries. This type of analysis, already presented on $\mathbf{5 0 0}$ units is here extended to more than 1000 completed units. The work also tries to identify which type of industry presents the learning percentages that are the most similar to our case and to investigate the impact of the production strategy on the process efficiency.
\end{abstract}

Index Terms-Accelerator magnets, production management.

\section{INTRODUCTION}

$\mathbf{T}$ HE Large Hadron Collider (LHC) is a $27 \mathrm{~km}$ circumference particle accelerator that is under construction at CERN, Geneva [1]. The accelerator is situated in a deep underground tunnel which is almost all filled with superconducting magnets [2] cooled at $1.9 \mathrm{~K}$ by means of superfluid helium [3]. The dipoles are the principal and most numerous of these magnets, determining the energy level of the particle beams: in total 1232 units, each $15 \mathrm{~m}$ long of 28 tonnes in a cold mass. The LHC dipole cold masses are being manufactured in three European industries [4], [5]: the consortium Alstom-Jeumont in France, Ansaldo Superconduttori in Italy, Babcock Nuclear Noell in Germany.

In the present work we try to analyse the production of the LHC Main Dipoles cold masses (Fig. 1) applying known techniques used in industrial production in order to estimate the "cost progress" or learning. The two terms describe the reduction in unit production cost as more units have been cumulatively produced over the course of a manufacturing program. This work is the update of a previous intermediate study [4]. For this update a total of 1125 collared coils and 1090 cold masses have been analysed. The authors refer to the previous analysis [4] for what concerns the general approach to the problem of the learning curves and the details related to the applied techniques. The aims of this analysis are:

The work of F. Regis was sponsored by ASP (Associazione per lo Sviluppo Tecnologico e Scientifico del Piemonte) and Compagnia di San Paolo, Turin, Italy.

The authors are with the Accelerator Technology Department, CERN, Geneva 23, 1211 CH, Switzerland (e-mail: paolo.fessia@cern.ch; federico. regis@cern.ch; lucio.rossi@cern.ch).

Digital Object Identifier 10.1109/TASC.2007.898521

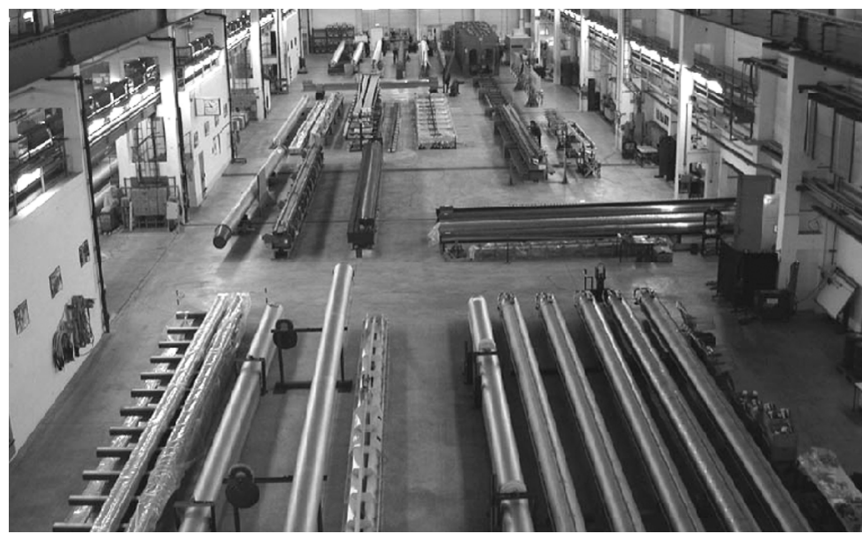

Fig. 1. LHC main dipole cold mass manufacturing at one of the 3 cold mass assemblers.

- Define what is the achievable limit in terms of cost progress for the LHC Main Dipole production and estimate the cost progress slope.

- Determine what is the upper limit until which the production can be scaled up (increase the production rate), with the given tooling, without reducing the efficiency, i.e., increase of unit cost.

- Quantify the learning slope describing the reduction in manufacturing labor hours and compare such value with other industries or similar productions.

The LHC Main Dipole production process can be divided in two parts. The first one, named Collared Coil (CC) production, implies the use of insulation and winding techniques and it can be classified in the category of special electrical machine assembly. The second one, hereunder called Cold Mass (CM) assembly, refers to the positioning of the CC inside the magnetic yoke and the enclosing of the whole in a mechanical structure which serves also as He II containment vessel. The CM assembly is based on precise heavy mechanics, requiring welding, alignment and vacuum technologies, as well as electric technologies at a simpler level than in the CC production. Due to the different technologies and consequently different personnel skills involved, in the two parts of the manufacture and also because of the geographical separation of the two assembly places in all suppliers, the two parts of the assembly will be treated separately.

\section{Application OF THE CRAWFORD AND WRight Models}

The 1st part of the analysis carried out is based on the application of two cost progress models widely used in industry [5]-[7]: 


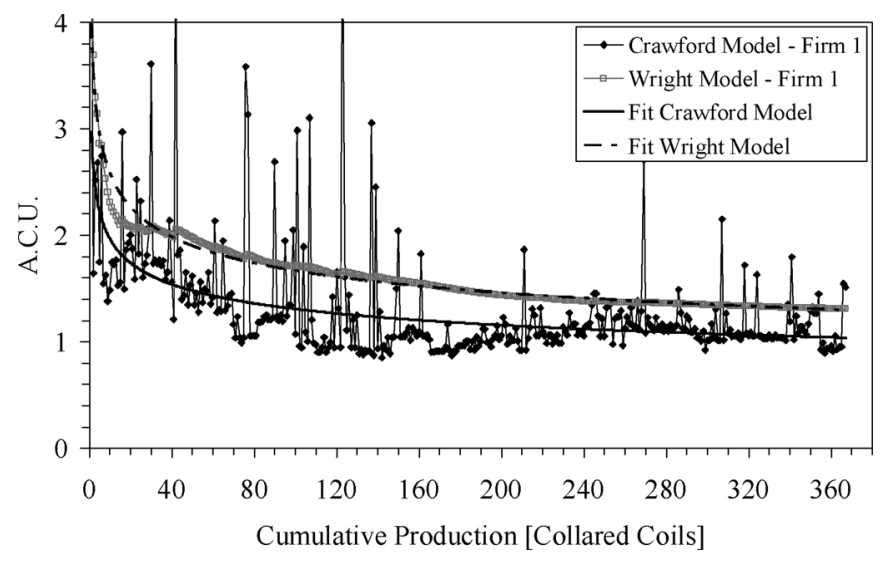

Fig. 2. Application of the Crawford and Wright models to collared coil production in Firm 1.

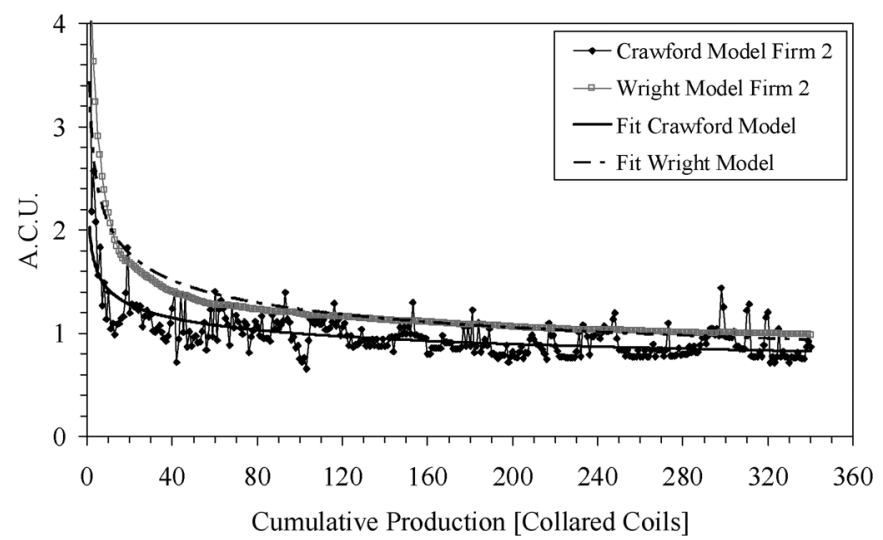

Fig. 3. Application of the Crawford and Wright models to collared coil production in Firm 2.

- the Crawford model based on the marginal cost of the unit $Q$ :

$$
M C(Q)=T_{1} Q^{b}
$$

The ratio of marginal cost for any two units depends only on their relative position in the manufacturing sequence.

- the Wright model, expressing the cumulative average cost of the first $Q$ units as a power function:

$$
A C(Q)=A_{1} Q^{\beta}
$$

The two models are theoretically the same for large samples and when non recurring costs equal to zero. When the latter are included in the model (as it is for LHC magnet production), it is better to use the first one. The learning percentage $\rho$ indicates the fraction to which is reduced the production cost per unit every time the number of completed units doubles (Crawford model):

$$
\rho=M C(2 Q) / M C(Q)=2^{b}
$$

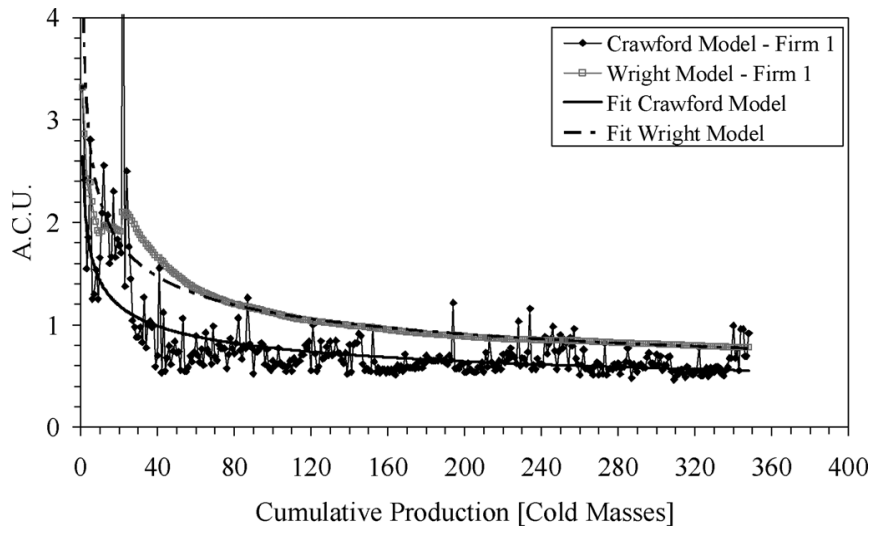

Fig. 4. Application of the Crawford and Wright models to cold mass production in Firm 1.

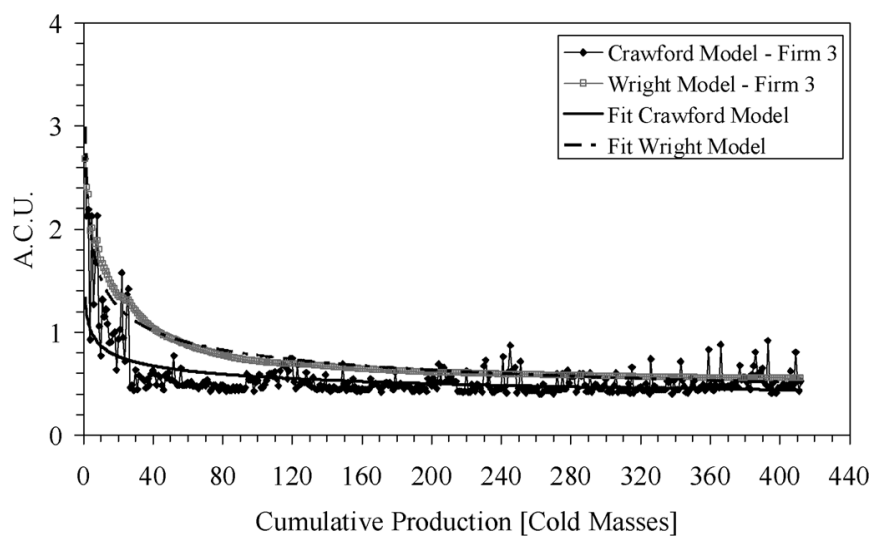

Fig. 5. Application of the Crawford and Wright models to cold mass production in Firm 3.

The higher is $\rho$ the lower is the learning improvement that have been reached. Figs. 2 and 3 show, for the first two firms, the evolution of the production cost of each assembled Collared Coil as function of the quantity of completed units. The costs of $\mathrm{CC}$ and $\mathrm{CM}$ have been normalized to the same arbitrary value for all the three firms. The analysis of the cost profiles indicates that the 3 firms present similar, but not equal final production costs. The spread is in any case quite small if we take into account the different cost structure linked to the differences in the labor market among the 3 nations where they are placed. In addition not all the companies own the premises where the assembly takes places. In order to have a clearer situation we can compare the cost of 300th unit produced: for Firm 2 and Firm 3 is about 0.8 arbitrary cost unit (A.C.U.), whereas it is about 1 A.C.U. for Firm 1.

In Figs. 4 and 5 the same plots as for the CC production are reported for the Cold Mass assembly (Firm 1 and Firm 3). In this case for the 300th produced unit the cost is 0.55 A.C.U for Firm 1 and 0.4 for Firm 2 and Firm 3. In both collared coil and cold mass production firm 1 results to be more "costly" compared to the other 2 companies by about $20 \%-30 \%$. Despite the very similar final cost is worth analysing how the 3 companies reached these values. Looking at the learning percentages $\rho$ (Tables I 
TABLE I

Learning Percentage AcCording to Crawford and Wright Models COLlaRed CoILS PRODUCTION

\begin{tabular}{lcc}
\hline \hline Firm & Crawford Model & Wright Model \\
\hline Firm 1 & $88 \%$ & $88 \%$ \\
Firm 2 & $90 \%$ & $86 \%$ \\
Firm 3 & $89 \%$ & $88 \%$ \\
\hline \hline
\end{tabular}

TABLE II

Learning Percentage AcCording to CRawford and Wright Models COLD MASS PRODUCTION

\begin{tabular}{lcc}
\hline \hline Firm & Crawford Model & Wright Model \\
\hline Firm 1 & $83 \%$ & $81 \%$ \\
Firm 2 & $82 \%$ & $81 \%$ \\
Firm 3 & $88 \%$ & $82 \%$ \\
\hline \hline
\end{tabular}

and II) the three companies present very similar values for both collared coil production and cold mass production.

It is worth to remark that

- With respect to the previously presented analysis the data are in very good agreement thanks to the very significant population analysed for the 3 companies. In addition the production history of each single unit has been studied in details to get rid of idle time (time spent by the unit in the workshop without activity), idle time that can change from company to company in function of the production organization.

- The Collared Coil production shows an average learning percentage higher by about 10 points with respect to the Cold Mass production learning percentage and therefore a lower learning capacity. This feature is very likely linked to the necessity of several and delicate test phases during the collaring phase, such as: electrical and dimensional checks on the inner and outer layers after curing, on each pole after assembling the layers as well as on the collared coils at the end of the process, considering the magnetic test as the last step of the manufacture. In addition these steps cannot be shortened without impairing the final quality product.

\section{RELATIONSHIP BETWEEN UNIT INCREMENTAL COST, MANPOWER EMPLOYED AND WeEKLY PRODUCTION RATE}

In order to evaluate the limitations of the production process necessary to assemble an LHC Main Dipole, it is important to check if the process itself can be scaled up in size. This will indicate also if the main assembly tool provided by CERN is a limiting factor in the cost progress. If this is the case over a defined production rate an addition of personnel would not generate a proportional increase in output. An increase of the unit incremental costs could also appear because more manpower would be divided among the same numbers of assembled units showing that the production becomes inefficient.

The analysis is focusing here on the second phase of the manufacture, the Cold Mass assembly. For this process the quantity and type of main tooling installed at the three firms is identical and the production differs only for the choices made by each company concerning the production logistics, production flow, internal organization and management of the staff. Comparing

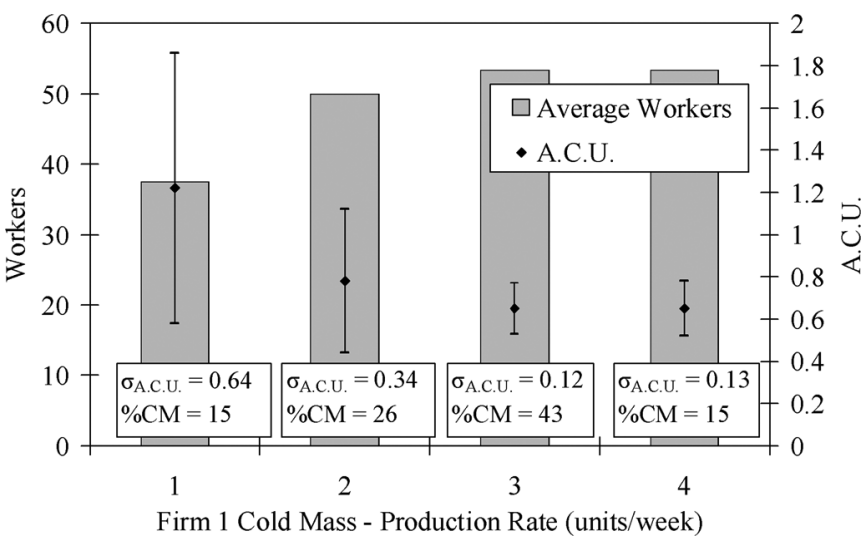

Fig. 6. Incremental cost of produced units respect to number of average workers employed and weekly production rates. Firm 1.

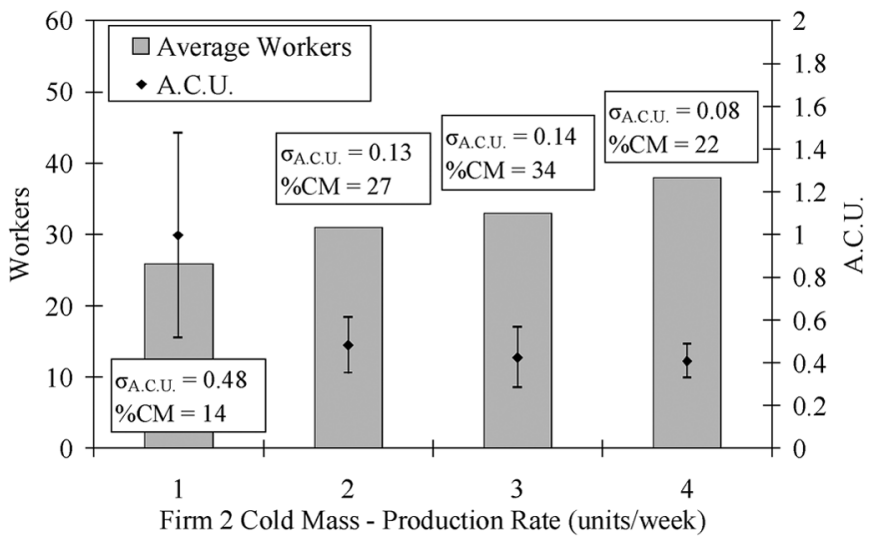

Fig. 7. Incremental cost of produced units respect to number of average workers employed and weekly production rates. Firm 2.

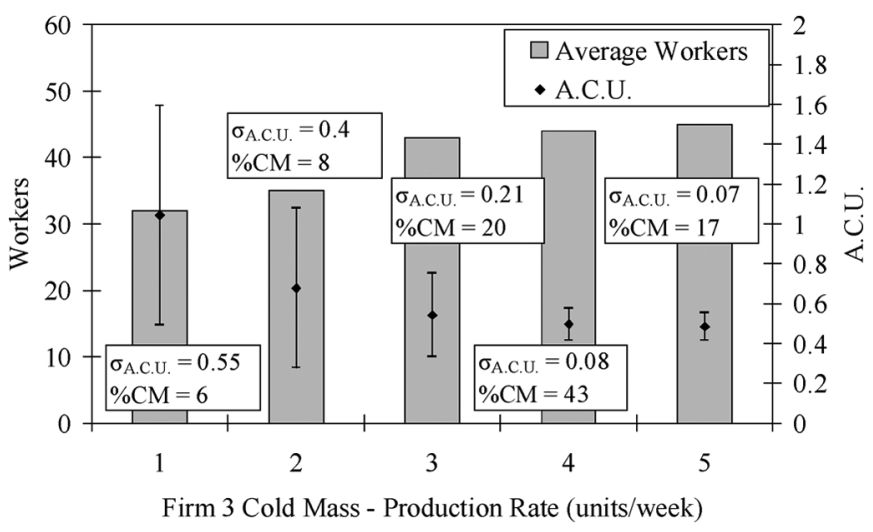

Fig. 8. Incremental cost of produced units respect to number of average workers employed and weekly production rates. Firm 3.

the results among the three firms (Fig. 6, Fig. 7, and Fig. 8) it is possible to draw the following conclusions:

- The CERN-provided tooling is not limiting the production rate till a value of 5-6 units/week. With adequate organization all the production phases are scalable at least to a level of 4-5 units/week.

- Higher production rates normally match lower production costs. The limits seem to be 4 units/week for Firm 1 and Firm 2 and 5 unit/week for Firm 3 (the number of workers 
TABLE III

LEARNING PERCENTAge ON MAN Hours EMPLOYed

\begin{tabular}{lcc}
\hline \hline Firm & Collared Coil & Cold Mass \\
\hline Firm 1 & $81 \%$ & $82 \%$ \\
Firm 2 & $88 \%$ & $80 \%$ \\
Firm 3 & $82 \%$ & $83 \%$ \\
\hline \hline
\end{tabular}

TABLE IV

LEARNING PERCENTAGE OF SELECTEd REFERENCE INDUSTRIES

\begin{tabular}{lc}
\hline \hline \multicolumn{1}{c}{ Industry } & $\rho$ \\
\hline Complex machine tools for new models & $75 \%-85 \%$ \\
Repetitive electrical operations & $75 \%-85 \%$ \\
LHC magnets & $80 \%-85 \%$ \\
Shipbuilding & $80 \%-85 \%$ \\
Aerospace & $85 \%$ \\
Purchased Parts & $85 \%-88 \%$ \\
Repetitive welding operations & $90 \%$ \\
Repetitive electronics manufacturing & $90 \%-95 \%$ \\
Repetitive machining or punch-press operations & $90 \%-95 \%$ \\
Raw materials & $93 \%-96 \%$ \\
\hline \hline
\end{tabular}

shown has been averaged on the number of magnets corresponding to that production rate). The graphs report also which percentage of the completed production has been produced with a certain rate. No correlation could be noticed between the highest production rates and loss of quality in the final product

Similar observations are feasible also for the processes related to the collared coil assembly with the remarks that Firm 2 and Firm 3 reach the minimum cost for a production rate of $4 \mathrm{CC} /$ week while for Firm 1 the optimum is reached for a production rate of $3 \mathrm{CC} /$ week and this is linked to its smaller installed winding capacity.

\section{LEARNing IN TERM OF Reduction of Production Time}

In order to evaluate the reduction in time necessary to complete and assembly we will use the learning percentage $\rho$. The value of $b$ (see (1)) is deduced by fitting the available production data with a simple potential relation. The results, in terms of learning percentage, are reported in Table III.

We can estimate that the typical learning percentage for the LHC Main Dipole Assembly is between $80 \%$ and $85 \%$.This value is lower than previously estimated on a smaller set of data in the first study. During RHIC construction the estimation of similar parameters provided a value of $85 \%$ [10] (Table IV). Fundamental and successful has been the choice to divide the whole production among three different firms, allowing to reach a level of learning comparable to repetitive electrical operations and shipbuilding.

\section{CONCLUSIONS}

The learning curve analysis has been applied to the $80 \%$ of the production of the main dipoles for the LHC. The results show that with respect to the previous analysis the learning percentage are in reality higher, indicating a lower learning speed. Nevertheless the results position this production among the fastest learning industries. The analysis confirms also that the main tooling installed is not a limiting factor and that the choice of having three suppliers was actually very good, in terms of production time, delivery to CERN and reached learning percentage. This choice was also instrumental in pursuing the cost minimization, keeping the three firms in competition among each others. It is worth pointing out that the largest part of the learning is consolidated after 30-40 completed units underlining the importance of having a 2 phase contract in order to better optimize costs and prices. In the present analysis, the very important reduction of production time during this phase is more pronounced with respect to the previous analysis [4]. This is also linked to the way in which the data have been treated, eliminating the idle time for those magnets that have been reworked.

\section{ACKNOWLEDGMENT}

The authors thank Mr. D. Bresson (Alstom MSA), Mr. J.F. De Coene (Jeumont Industries), Mr. P. Gagliardi (Ansaldo Superconduttori) and Mr. W. Gaertner and Mr. P. Lublow (Babcock Nuclear Noell) and their staff for providing essential data for this analysis. A warm thanks also to the CERN-ISQ resident inspectors for the data collection. In addition we would like to thank also the whole CERN team in charge of the follow up of the production of the LHC Main Dipoles.

\section{REFERENCES}

[1] O. Bruning, LHC Design Report: The LHC Main Ring CERN-2004003 , vol. I.

[2] L. Rossi, "State-of-the-art superconducting accelerator magnets," IEEE Trans. Appl. Supercond., vol. 12, no. 1, pp. 219-227, March 2002.

[3] L. Tavian, "Latest developments in cryogenics at CERN," presented at the 20th National Symposium on Cryogenics-2005 TNSC 2005, Surat, India, Feb. 24-26, 2005, unpublished.

[4] P. Fessia, L. Rossi, and S. Krog-Pedersen, "Application of the learning curve analysis to the LHC main dipole production: First assessment," IEEE Trans. Appl. Supercond., vol. 16, no. 2, pp. 242-247, June 2006.

[5] L. Rossi, "The LHC main dipoles and quadrupoles toward series production," IEEE Trans. Appl. Supercond., vol. 13, no. 2, pp. 1221-1228, June 2003.

[6] M. S. Goldberg and A. E. Touw, Statistical Methods for Learning Curves and Cost Analyses. : Military Applications Society, 2003, 1-877640-18-2.

[7] G. Li and S. Rajagopalan, "The impact of quality on learning," Journal of Operations Management, vol. 15, pp. 181-191, 1997.

[8] R. S. Russel and B. W. Taylor, Operations Management, 4th ed. : Prentice Hall, 2003, 0-13-034834-1.

[9] M. D. Anerella and D. H. Fischer, "Industrial production of RHIC magnets," IEEE Trans. Magnetics, vol. 32, no. 4, July 1996.

[10] D. Fischer, M. Anerella, and P. Wanderer, "Successful partnership between Brookhaven National Laboratory and Northtrop Grumman Corp. for construction of RHIC superconducting magnets," IEEE Trans. Superconductivity, vol. 10, no. 1, March 2000. 